Literature research shows that infusion of noradrenalin and dobutamine before induction is effective in countering the depressive effect of anesthetic drugs. ${ }^{6}$ Further, cardiostable and short-acting drugs such as etomidate, fentanyl, and vecuronium are employed as part of anesthetic regime. Noninvasive continuous cardiac output monitoring is used to evaluate the ventricular performance in response to fluid therapy, inotropes, and prone positioning. ${ }^{7}$ Prone position leads to increased intrathoracic pressure reducing venous return, and decrease in cardiac output (CO) and cardiac index (CI), which is exaggerated in DCM patients.

A noteworthy point with regard to pacing in these patients: preoperative conversion to asynchronous mode of CRT-P is necessary, in order to prevent pacemaker inhibition by electromechanical interference and use of bipolar cautery and also prevent pacemaker malfunction. For AICD, since these patients are not pacemaker-dependent, simple deactivation of the ICD intraoperatively may suffice. ${ }^{8,9}$ Additionally, AED pads should be placed before induction in the anterior-posterior configuration, so that the cardioversion/defibrillation current would not cross the pacemaker path.

\section{Conclusion}

Anesthetic management of patients with DCM on CRT device poses unique challenge for the anesthesiologist, with strong likelihood of catastrophic hemodynamic perturbations, dysrhythmias, and even sudden cardiac arrest. Particularly in surgery in prone position with limited access to the patient in the event of a catastrophe, meticulous planning, vigilant monitoring, adequate preparation with anterior-posterior configuration of transthoracic pads, judicious use of pharmacological agents, and tailor-made anesthetic regime, can lead to a favorable outcome.

\section{Conflict of Interest}

None declared.

\section{References}

1 Kaur H, Khetarpal R, Aggarwal S. Dilated cardiomyopathy: an anaesthetic challenge. J Clin Diagn Res 2013;7(6):1174-1176

2 Pradhan R, Vaidya PR, Shrestha RR, Parajuli S, Joshi HR, Shah BL. Dilated cardiomyopathy and anesthesia in prone position: a case report. Postgraduate Medical Journal of NAMS. 2016;16:42-45

3 Raj R, Kumar M, Batra M. Anaesthetic management of a case of dilated cardiomyopathy for emergency appendectomy. Anesth Essays Res 2014;8(1):105-107

4 Zhou Y, Liu L, Cheng T, et al. Grade 3 echocardiographic diastolic dysfunction is associated with increased risk of major adverse cardiovascular events after surgery: a retrospective cohort study. Anesth Analg 2019;129(3):651-658

5 Godfrey GEP, Peck MJE. Diastolic dysfunction in anaesthesia and critical care. BJA Educ 2016;16(9):287-291

6 Davies MR, Cousins J. Cardiomyopathy and anaesthesia. Contin Educ Anaesth Crit Care Pain 2009;9(6):189-193

7 Alhashemi JA, Cecconi M, Hofer CK. Cardiac output monitoring: an integrative perspective. Crit Care 2011;15(2):214

8 Stone ME, Salter B, Fischer A. Perioperative management of patients with cardiac implantable electronic devices. $\mathrm{Br} \mathrm{J}$ Anaesth 2011;107(Suppl 1):i16-i26

9 Practice Advisory for the Perioperative Management of Patients with Cardiac Implantable Electronic Devices: Pacemakers and Implantable Cardioverter-Defibrillators 2020: An Updated Report by the American Society of Anesthesiologists Task Force on Perioperative Management of Patients with Cardiac Implantable Electronic Devices*. Anesthesiology 2020;132(2): 225-252

\title{
Management of a Difficult Airway Scenario in a Case of Hurler's Syndrome with a D-Blade Video Laryngoscope
}

\author{
Keta D. Thakkar ${ }^{1, \odot}$ Ajay P. Hrishi ${ }^{1, \odot} \quad$ Manikandan Sethuraman ${ }^{1, \odot} \quad$ Smita Vimala ${ }^{1, \odot}$ \\ ${ }^{1}$ Department of Anaesthesiology, Division of Neuroanesthesia, \\ Sree Chitra Tirunal Institute for Medical Sciences and Technology, \\ Thiruvananthapuram, Kerala, India \\ Address for correspondence Ajay P. Hrishi, MD, DM, Department \\ of Anaesthesiology, Division of Neuroanesthesia, Surgical block, \\ Sree Chitra Tirunal Institute for Medical Sciences and Technology, \\ Trivandrum, Kerala 695011, India (e-mail: drajay@sctimst.ac.in).
}

J Neuroanaesthesiol Crit Care 2021;8:214-216.

published online

August 10, 2020
DOI https://doi.org/

$10.1055 / \mathrm{s}-0040-1714451$

ISSN 2348-0548. (c) 2020. Indian Society of Neuroanaesthesiology and Critical Care. This is an open access article published by Thieme under the terms of the Creative Commons Attribution-NonDerivative-NonCommercial-License, permitting copying and reproduction so long as the original work is given appropriate credit. Contents may not be used for commercial purposes, or adapted, remixed, transformed or built upon. (https://creativecommons.org/licenses/by-nc-nd/4.0/).

Thieme Medical and Scientific Publishers Pvt. Ltd. A-12, 2nd Floor, Sector 2, Noida-201301 UP, India 


\begin{abstract}
Keywords

- Hurler's syndrome

- pediatric difficult airway

- D-blade C-MAC

Hurler's syndrome is a rare genetic disease characterized by progressive multiorgan accumulation of glycosaminoglycans. It is associated with progressive craniofacial, skeletal, and cardiac involvement, which increases the risk of anesthesia. Patients with Hurler's syndrome could present as the worst airway management problem an anesthesiologist could deal with due to abnormal upper airway anatomy and limited neck manipulation, owing to the atlantoaxial instability. We report a case of difficult airway scenario of a child with Hurler's syndrome, leading to an apparent cannot ventilate cannot intubate scenario, which was managed successfully with the help of a C-MAC video laryngoscope with unique D-blade. In Hurler's syndrome, C-MAC with D-blade is an excellent tool in establishing an airway in a pediatric difficult airway scenario. Moreover, D-blade C-MAC could be considered as the primary tool for establishing an airway in pediatric patients with Hurler's syndrome.
\end{abstract}

\section{Introduction}

Hurler's syndrome, a type 1 mucopolysaccharidosis (MPS 1H), is an uncommon genetic disease caused by deficiency of a specific lysosomal enzyme, a-1-iduronidase, which is required for a normal degradation of glycosaminoglycans and characterized by progressive multiorgan accumulation of the same. ${ }^{1}$ It is typically associated with progressive craniofacial, skeletal, and cardiac involvement, which increases the risk of anesthesia. ${ }^{1,2}$ We report a case of a difficult airway scenario of a child with Hurler's syndrome, leading to an apparent cannot ventilate cannot intubate scenario, managed successfully with the help of a C-MAC video laryngoscope and pediatric D-blade. Informed consent was obtained from the parents prior to submission for publication.

\section{Case Report}

A 2-year-old girl, with developmental delay and history of focal epileptic seizures, presented to the MRI suite for a diagnostic scan. At about 1 month after normal birth, she was noted to have a short neck and restricted movements of shoulders and wrists. The mother gave a history of snoring during sleep with a protruded tongue. On examination, she had short stature with frontal bossing and short neck. She was uncooperative with regard to further airway examination, but difficult airway was anticipated owing to the history of obstructive sleep apnea and large head with restricted neck movement. The patient's previous medical documents revealed that she is a known case of Hurler's syndrome and hence a difficult airway cart was kept ready before induction. Informed and written consent for surgical airway was obtained and a surgeon was kept standby in view of anticipated difficult airway. The patient was induced with a high-concentration primed circuit technique with $8 \%$ Sevoflurane, and 24G intravenous access was secured. The head was positioned for intubation, and a shoulder roll was inserted. Depth of anesthesia was deepened with propofol $0.5 \mathrm{mg} \cdot \mathrm{kg}^{-1}$, and direct laryngoscopy (DL) was attempted, which revealed a Cormack-Lehane (CL) grade IV with tonsillar hypertrophy (grade 3). An attempt of blind intubation with the help of a pediatric bougie was made and was unsuccessful. Following this episode, the mask ventilation was also proving to be complicated, even after optimizing the head position and the oropharyngeal airway. Additionally, $1 \mathrm{mg} \cdot \mathrm{kg}^{-1}$ of propofol was administered, and attempts with laryngeal mask airway (LMA) size 1.5 and 2 also failed to ventilate, and the patient started desaturating, leading to an apparent cannot ventilate cannot intubate scenario.

The surgeon was intimated to secure a surgical airway and, at the same time, an attempt was made to ventilate with a face mask after removal of LMA. In the meanwhile, we decided on a trial laryngoscopy with pediatric D-blade and C-MAC (Karl Storz, Tuttlingen, Germany) video laryngoscope, and a CL grade I was visualized on video laryngoscopy. The airway was secured with a 3.5 size uncuffed endotracheal tube (ETT) railroaded on the stylet. After ensuring bilateral air entry, ETT was fixed, and the child was taken for MRI. The MRI revealed macrocephaly, large tongue, receding chin, and micrognathia. The MRI (T2 sagittal) revealed adenoid hypertrophy, leading to nasopharyngeal narrowing, which can explain the symptoms of snoring. The foramen magnum was narrowed due to ligamental hypertrophy ( - Fig. 1 ). Postprocedure, once the child awakened, the airway was extubated after ruling out airway edema and was observed in a high-dependency unit for 24 hours.

\section{Discussion}

Children with Hurler's syndrome can present with abnormal upper airway anatomy, poor thoracic compliance, and atlantoaxial instability. ${ }^{1,3}$ Unsuccessful intubation attempts have also resulted in airway-related deaths of children having mucopolysaccharidoses. ${ }^{4}$ Dealing with such a challenging scenario at a remote location outside operating rooms is a trying ordeal for an anesthesiologist.

The difficult airway encountered in Hurler's syndrome is attributed to the presence of macrocephaly, macroglossia, odontoid hypoplasia, dental distortions, gingival hyperplasia, swollen epiglottis, tonsillar enlargement, and anteriorization of the larynx. ${ }^{2,5}$ Face mask ventilation could be difficult and an adequate seal can be achieved with the help of an inverted mask technique. ${ }^{5}$ Narrowing of laryngeal and tracheobronchial 


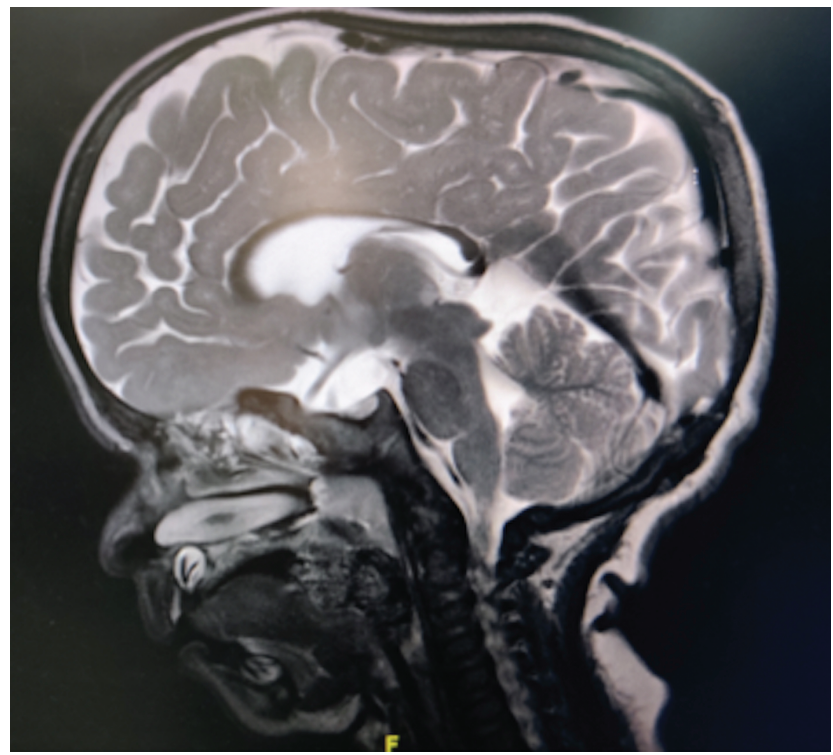

Fig. 1 MRI Head (T2 sagittal): of the patient with Hurler's syndrome showing macrocephaly, macroglossia, receding chin and micrognathia. MRI (T2 sagittal) revealed adenoid hypertrophy, leading to nasopharyngeal narrowing, which can explain the symptoms of snoring. The foramen magnum was narrowed due to ligamental hypertrophy.

cartilage often necessitates the need for a smaller-sized ETT. ${ }^{1}$ In our case, we secured the airway with a 3.5 size tube rather than in accordance with the appropriate for age formula, due to apparently smaller glottis which can be attributed to the deposition of glycosaminoglycans in the airway. We were able to ventilate without any leak. Due to the anticipated difficult airway in the majority of the patients, blind intubation can be tried, ${ }^{1}$ but in our case, we were not able to secure the airway with bougie-assisted blind oral intubation. Although LMA is useful in an emergency scenario in establishing a successful airway in some patients, it failed to guarantee adequate ventilation in our patient. A case report by Busoni et al, which described how LMA could lead to inspiratory stridor and airway obstruction in mucopolysaccharidosis II (Hunter syndrome), has been published, and these findings can be extrapolated for our patient due to resemblance in airway morphology. ${ }^{6}$ Hurler's syndrome is known to cause thick secretions in the airway and collapse of supraglottis after anesthesia, which could have inhibited effective ventilation in our patient at a later stage, even with an LMA. ${ }^{1}$

In our scenario, we succeeded in securing the airway in the first attempt with the C-MAC pediatric D-blade and the head in the neutral position. Pediatric D-blade has been specially designed for difficult airway with CL III or IV and an acutely angulated design, giving it a half-moon shape. ${ }^{7}$ The degree of angulation is 40 compared with 18 with a conventional C-MAC Mackintosh blade. The neutral position of the head is ideal due to the high-incidence of odontoid dysplasia in this group of patients, which could be ensured with the help of this unique video laryngoscope. Although the DAS pediatric difficult airway guidelines advocate the percutaneous cricothyroidotomy in cannot ventilate cannot intubate scenario after repeated attempts with LMA, C-MAC with a pediatric D-blade could be considered as a noninvasive and appealing option for these patients. ${ }^{8}$

\section{Conclusion}

In Hurler's syndrome, C-MAC with D-blade is an excellent tool in establishing an airway in a pediatric difficult airway scenario. Moreover, D-blade C-MAC could be considered as the primary tool for establishing an airway in pediatric patients with Hurler's syndrome.

\section{Conflict of Interest}

None declared.

\section{References}

1 Spinello CM, Novello LM, Pitino S, et al. Anesthetic management in mucopolysaccharidoses. ISRN Anesthesiol 2013. Doi: $10.1155 / 2013 / 791983$

2 Clark BM, Sprung J, Weingarten TN, Warner ME. Anesthesia for patients with mucopolysaccharidoses: Comprehensive review of the literature with emphasis on airway management. Bosn J Basic Med Sci 2018;18(1):1-7

3 Gupta AK, Kharde V, Gawer V, Sidhaye RV, Divekar DS. Hurler's syndrome: anaesthetic challenges and management. Anestesia Pediatrica e Neonatale 2010;8(2):1-8

4 Nicolson SC, Black AE, Kraras CM. Management of a difficult airway in a patient with Hurler-Scheie syndrome during cardiac surgery. Anesth Analg 1992;75(5):830-832

5 Gupta N, Rath GP, Bala R, Reddy BK, Chaturvedi A. Anesthetic management in children with Hurler's syndrome undergoing emergency ventriculoperitoneal shunt surgery. Saudi J Anaesth 2012;6(2):178-180

6 Busoni P, Fognani G. Failure of the laryngeal mask to secure the airway in a patient with Hunter's syndrome (mucopolysaccharidosis type II) Paediatr Anaesth 1999;9(2):153-155

7 Kilıçaslan A, Topal A, Erol A, Uzun ST, Tuncer S, Uzen ST. Comparison of the C-MAC D-Blade, conventional C-MAC, and Macintosh laryngoscopes in simulated easy and difficult airways. Turk J Anaesthesiol Reanim 2014;42(4):182-189

8 Frerk C, Mitchell VS, McNarry AF, et al; Difficult Airway Society intubation guidelines working group. Difficult Airway Society 2015 guidelines for management of unanticipated difficult intubation in adults. Br J Anaesth 2015;115(6):827-848 